\title{
Comprehensive Imaging of Gastroesophageal Biopsy Samples by Spectrally Encoded Confocal Microscopy
}

\section{Citation}

Kang, DongKyun, Melissa J. Suter, Caroline Boudoux, Hongki Yoo, Patrick S. Yachimski, William P. Puricelli, Norman S. Nishioka, et al. 2010. Comprehensive imaging of gastroesophageal biopsy samples by spectrally encoded confocal microscopy. Gastrointestinal Endoscopy 71(1): 35-43. doi:10.1016/j.gie.2009.08.026

\section{Published Version}

doi:10.1016/j.gie.2009.08.026

\section{Permanent link}

http://nrs.harvard.edu/urn-3:HUL.InstRepos:32543164

\section{Terms of Use}

This article was downloaded from Harvard University's DASH repository, and is made available under the terms and conditions applicable to Other Posted Material, as set forth at http:// nrs.harvard.edu/urn-3:HUL.InstRepos:dash.current.terms-of-use\#LAA

\section{Share Your Story}

The Harvard community has made this article openly available.

Please share how this access benefits you. Submit a story.

\section{Accessibility}




\title{
Comprehensive imaging of gastroesophageal biopsy samples by spectrally encoded confocal microscopy
}

\author{
DongKyun Kang, PhD, Melissa J. Suter, PhD, Caroline Boudoux, PhD, Hongki Yoo, PhD, \\ Patrick S. Yachimski, MD, William P. Puricelli, RN, Norman S. Nishioka, MD, Mari Mino- \\ Kenudson, MD, Gregory Y. Lauwers, MD, Brett E. Bouma, PhD, and Guillermo J. Tearney, \\ MD, PhD \\ Boston, Massachusetts, USA
}

\section{Abstract}

Background-Spectrally encoded confocal microscopy (SECM) is a high-speed reflectance confocal microscopy technique that has the potential to be used for acquiring comprehensive images of the entire distal esophagus endoscopically with subcellular resolution.

Objective-The goal of this study was to demonstrate large-area SECM in upper GI tissues and to determine whether the images contain microstructural information that is useful for pathologic diagnosis.

Design-A feasibility study.

Setting-Gastrointestinal Unit, Massachusetts General Hospital.

Patients-Fifty biopsy samples from 36 patients undergoing routine EGD were imaged by SECM, in their entirety, immediately after their removal.

Results-The microstructure seen in the SECM images was similar to that seen by histopathology. Gastric cardia mucosa was clearly differentiated from squamous mucosa. Gastric fundic/body type mucosa showed more tightly packed glands than gastric cardia mucosa. Fundic gland polyps showed cystically dilated glands lined with cuboidal epithelium. The presence of intraepithelial eosinophils was detected with the cells demonstrating a characteristic bilobed nucleus. Specialized intestinal metaplasia was identified by columnar epithelium and the presence of goblet cells. Barrett's esophagus (BE) with dysplasia was differentiated from specialized intestinal metaplasia by the loss of nuclear polarity and disorganized glandular architecture.

Limitations-Ex vivo, descriptive study.

Conclusions-Large-area SECM images of gastroesophageal biopsy samples enabled the visualization of both subcellular and architectural features of various upper GI mucosal types and were similar to the corresponding histopathologic slides. These results suggest that the development of an endoscopic SECM probe is merited.

Copyright (C) 2010 by the American Society for Gastrointestinal Endoscopy

Reprint requests: Guillermo J. Tearney, MD, PhD, Harvard Medical School, Massachusetts General Hospital, and Wellman Center for Photomedicine, 40 Blossom Street, BAR 703, Boston, MA 02114.

Current affiliations: Wellman Center for Photomedicine (D.K., M.J.S., H.Y., B.E.B., G.J.T.), Department of Dermatology (D.K.,

M.J.S., H.Y., B.E.B.), Gastrointestinal Unit (W.P.P., N.S.N.), and Department of Pathology (M.M.K., G.Y.L., G.J.T.), Harvard

Medical School, Massachusetts General Hospital, Boston, Massachusetts, Department of Engineering Physics (C.B.), Ecole

Polytechnique Montreal, Montreal, Quebec, Canada, Division of Gastroenterology, Hepatology, and Nutrition (P.S.Y.), Vanderbilt

University Medical Center, Nashville, Tennessee, USA.

DISCLOSURE: This research was supported by National Institutes of Health/National Cancer Institute (grant number

R21CA122161). All authors disclosed no financial relationships relevant to this publication. 
The diagnosis of esophageal diseases is hampered by the inability of conventional video endoscopy to visualize pathologies such as dysplasia and early cancer in patients with Barrett's esophagus (BE) and eosinophilic esophagitis. Many approaches are being investigated to address this issue, including endoscopic microscopy or endomicroscopy, where high-resolution, microscopic images are acquired in vivo by using an endoscopic probe. ${ }^{1,2}$ Confocal endomicroscopy is one technology that is capable of providing images that contain cellular and subcellular features of the esophagus. ${ }^{3,4}$ In previous reports, confocal endomicroscopy has been shown to distinguish squamous mucosa from gastric pits, ${ }^{5} \mathrm{BE}$ with specialized intestinal metaplasia (SIM) from normal gastric or squamous epithelium, ${ }^{6,7}$ and neoplasia associated with BE from SIM. ${ }^{6-8}$

Although confocal endomicroscopy shows promise, its field size is usually limited to less than $500 \mu \mathrm{m} .{ }^{5,9}$ Thus, confocal endoscopic imaging may be subject to sampling errors similar to those of endoscopic biopsy. ${ }^{10}$ Mosaicing, or stitching, of multiple adjacent confocal images has been demonstrated to generate a considerably larger imaging area, ${ }^{9,11}$ but even with these techniques, the total attainable field size is often significantly smaller than that of the mucosal surface area at risk, which can have a longitudinal extent of many centimeters. ${ }^{12}$ A different form of endomicroscopy, termed comprehensive volumetric microscopy, has been proposed that acquires a 3-dimensional microscopic image of the entire distal esophagus. This approach has been demonstrated by using high-speed optical frequency domain imaging (OFDI) through balloon-centering catheters. ${ }^{13,14}$ Although OFDI can provide architectural features of the imaged areas, cellular features are not well appreciated because of the 10 - to $20-\mu \mathrm{m}$ resolution of this technology.

An alternative reflectance imaging technology that has higher spatial resolution is reflectance confocal microscopy (RCM). RCM has been successfully used in imaging various types of human tissues, including esophageal, ${ }^{15,16}$ gastric, ${ }^{15,16}$ cervical, ${ }^{17,18}$ skin, ${ }^{19,20}$ joint, ${ }^{21}$ and breast tissues. ${ }^{22}$ In RCM, characteristic cellular and architectural features were rapidly visualized at video rates. ${ }^{16,18,20,23}$ Although the image quality demonstrated in these RCM studies is very good, frame rates need to be increased further to comprehensively screen large luminal organ surface areas in living patients.

Spectrally encoded confocal microscopy (SECM) is a high-speed RCM technique that is capable of obtaining images at very high speeds. ${ }^{24}$ With SECM, broadband or wavelengthswept narrowband light is coupled into a single optical fiber. Light from the fiber illuminates a grating and an objective lens at the distal end of the confocal probe. This configuration causes the sample to be illuminated at several different locations, where each point is interrogated by a different wavelength. After the light is transmitted back through the optics into the fiber, the image is reconstructed by detecting the returned light as a function of wavelength with a high-speed detector in the system's console. Because SECM does not need a rapid beam-scanning device inside the probe, the size of the optics can be small and the imaging speed can be very high, as much as 10 times faster than the video rate. These features of the SECM technology make comprehensive confocal endomicroscopy of the entire distal esophagus possible. The goal of this study was to investigate large-area SECM images of upper GI tissues and to provide a preliminary assessment as to whether these images contain sufficient microscopic information for the diagnosis of upper GI tract pathology.

\section{METHODS}

\section{SECM bench-top system}

A bench-top SECM system (Fig. 1) was used for the clinical study. A wavelength-swept laser (central wavelength $=1320 \mathrm{~nm}$; bandwidth $=70 \mathrm{~nm}$; repetition rate $=5 \mathrm{kHz})^{25}$ was 
used as the light source. A transmission grating (groove density $=1100$ lines $/ \mathrm{mm}$ ) and a water-immersion objective lens (effective numerical aperture $=0.7$; focal length $=5.3 \mathrm{~mm}$ ) were used to generate a single-scan field length of $180 \mu \mathrm{m}$ along the spectrally encoded axis of the image. Two computer-controlled translational stages were used to scan the spectrally encoded line to obtain large-area images of biopsy samples. A piezoelectric transducer actuator was used to change the focal depth of the objective lens. The transverse resolution was measured to be $2 \mu \mathrm{m}$ and the axial resolution was $10 \mu \mathrm{m}$. The image dimensions varied from $2 \times 1 \mathrm{~mm}(4000 \times 2000$ pixels $)$ to $5 \times 3.6 \mathrm{~mm}(10,000 \times 7200$ pixels $)$. The number of axial (depth) sections varied from 8 to 15 with axial scan intervals ranging from 15 to 10 $\mu \mathrm{m}$, respectively, resulting in a total depth range of between 120 and $150 \mu \mathrm{m}$. The imaging time was between 2.5 and 15 minutes, depending on the biopsy sample size.

\section{Patient enrollment and imaging procedure}

Studies were conducted on biopsy samples taken from patients undergoing routine EGD at the Massachusetts General Hospital (MGH) Gastrointestinal Unit from June 2008 to February 2009. Any patient who underwent forceps biopsy was considered for enrollment, and biopsy samples from randomly selected sites from the esophagus and stomach were imaged. The biopsy samples were gently washed with saline solution. The first 14 biopsy samples were imaged in phosphate-buffered saline solution, and the remaining 36 samples were immersed in diluted acetic acid ( $0.6 \%$ concentration) to enhance nuclear contrast. The samples, with the epithelial surfaces preferably facing the objective lens, were placed under cover-slips and then imaged in their entirety by the SECM bench-top system at 8 to 15 depth levels. Imaging began less than 5 minutes after biopsy of the patient. After imaging, the acetic acid-stained samples were washed in saline solution to reverse the aceto-whitening. ${ }^{26}$ Each imaged sample was then placed in formalin and processed for paraffin-embedded histopathology by using standard methods. Hematoxylin and eosin (H\&E) histopathology was conducted per the standard of care at the MGH Gastrointestinal Pathology Service. Slides were digitized by using a full-slide scanner (ScanScope CS, Aperio Technologies, Inc, Vista, Calif). SECM readers and members of the MGH Gastrointestinal Pathology Service compared corresponding SECM and histopathology images. The SECM readers were informed of the histopathologic diagnoses before reading the corresponding SECM images. The study protocol was approved by the Partners Internal Review Board (IRB \#2007P000656).

\section{RESULTS}

Because it was difficult to orient the specimens grossly, of the 50 biopsy specimens imaged, 13 were positioned under the SECM microscope upside down. SECM images of the epithelium were not obtained for these 13 biopsy specimens because of the limited penetration depth of SECM through the submucosa. We therefore removed these 13 cases from the analysis. The remaining 37 biopsy samples were correctly oriented, 26 of which were stained by acetic acid. The diagnoses of the 37 biopsy samples are summarized in Table 1. Because the orientation of the samples was lost during the fixation and sectioning procedure, exact spatial correspondence between the SECM and histopathology images was not possible. For each mucosal type presented here, SECM and representative histopathology images obtained from the same biopsy specimen are shown at both low and high magnification.

\section{Gastroesophageal junctional mucosa}

Images of an acetic acid-stained biopsy sample taken at the gastroesophageal junction of a 46-year-old male patient with a history of BE with dysplasia are shown in Figure 2. The wide-field SECM image (Fig. 2A) enables the differentiation of gastric cardia type from 
squamous esophageal mucosa, based on architecture, and is similar to the low-power histopathology image (Fig. 2B). At higher power, both squamous and columnar cell nuclei can be identified by SECM (Fig. 2C). The histopathologic diagnosis for this case was squamoglandular junctional mucosa with focal intestinal metaplasia confirmed by the corresponding high-power histopathologic image (Fig. 2D).

\section{Gastric body/fundic type mucosa}

The images in Figure 3 were obtained from an acetic acid-stained biopsy sample taken from a 57-year-old female patient with a history of BE with dysplasia. The large-area SECM image (Fig. 3A) identifies closely packed glands. The histopathologic image (Fig. 3B) also confirms the presence of tightly packed glands of the gastric fundic/body type but also shows gastric foveolar epithelium. The high-magnification SECM image (Fig. 3C) occasionally shows the nuclei on the epithelia of the glands. The biopsy sample was diagnosed as gastric body/fundic type mucosa and gastric cardia type mucosa with acute and chronic inflammation.

\section{Fundic gland polyp}

Images of an acetic acid-stained biopsy sample from a gastric polyp of a 71-year-old female patient with a history of BE and gastric polyps are shown in Figure 4. The lowmagnification SECM image (Fig. 4A) depicts irregular glandular architecture, which is consistent with the low-magnification image of the corresponding H\&E-stained section (Fig. 4B). The high-magnification SECM image (Fig. 4C) shows cystically dilated glands with irregular sizes and shapes. The histopathologic images (Fig. 4B and D) exhibit gastric body/ fundic type mucosa with scattered cystically dilated glands consistent with a fundic gland polyp. The histopathologic diagnosis was also fundic gland polyp.

\section{Eosinophils}

Figure 5 shows images of a biopsy sample from the mid-esophagus of a 31-year-old patient with a history of eosinophilic esophagitis. In the large-area SECM image (Fig. 5A), several scattered white dots are observed. The magnified SECM image (Fig. 5C) of a white dot (Fig. $5 \mathrm{~A}$ ) reveals the distinctive bilobed nucleus of an eosinophil and highly scattering cytoplasm. The high-magnification histology image (Fig. 5D) also reveals an eosinophil with a bilobed nucleus. The sample was diagnosed as esophageal squamous mucosa with scattered eosinophils.

\section{SIM}

In Figure 6, images of an acetic acid-stained biopsy sample taken from a 76-year-old male patient with a history of BE with high-grade dysplasia (HGD) treated with photodynamic therapy are shown. The large-area SECM image (Fig. 6A) shows columnar epithelium distinguished from squamous epithelium. At higher magnification, the SECM image (Fig. 6C) reveals the presence of goblet cells, which is indicative of BE with SIM. Histopathology (Fig. 6B and D) confirmed the presence of intestinal-type mucosa with scattered goblet cells. For the 6 biopsy samples diagnosed as BE with no dysplasia, all the SECM image sets showed foveolar epithelium. Goblet cells were detected in 5 of 6 SIM biopsy samples. For the SIM case in which SECM did not reveal goblet cells, the histopathology showed that the intestinal metaplasia was buried, indicating that the intestinal metaplastic glands were located deeper than SECM interrogated.

\section{Dysplasia}

Figure 7 depicts images of an acetic acid-stained biopsy sample taken from a 75-year-old female patient with a history of BE with HGD and intramucosal adenocarcinoma treated 
with EMR, photodynamic therapy, the BARRX HALO 90 system (BARRX Medical, Inc, Sunnyvale, Calif) and cryotherapy. The wide-field SECM image (Fig. 7A) shows disorganized glands with irregular shapes and sizes without stroma compared with more regularly arranged glands with intervening stroma. The low-magnification histopathologic image (Fig. 7B) shows dysplastic surface epithelium and normal-appearing glands. On higher magnification, the SECM image (Fig. 7C) reveals the loss of nuclear polarity in epithelial cells and limited stratification of the nuclei that are confirmed by the corresponding histopathologic image (Fig. 7D). The histopathologic diagnosis was glandular mucosa with HGD.

\section{DISCUSSION}

Our results show that large-area SECM can reveal both of the architectural and cellular features of various gastroesophageal tissues, which are similar to the morphologic features seen on the corresponding H\&E-stained slides. Of particular importance is the ability of SECM to enable the visualization of goblet cells, nuclear stratification in dysplasia, and eosinophils.

There were several limitations of this study. First, the images were not acquired in vivo, but within 15 minutes after removal of samples by forceps biopsy. How tissue microstructure changes over this brief time period is not known. However, we are encouraged by other reports of RCM in vivo ${ }^{16}$ that have shown some of the features that we have seen in our biopsy study.

In this preliminary work, we applied acetic acid in the majority of cases to enhance nuclear contrast. This is a well-established technique for the esophagus 27,28 and other organ systems. ${ }^{29,30}$ For most of the gastroesophageal mucosal types examined in this study, images obtained with acetic acid visualized cell nuclei more reliably and with greater contrast than images obtained without acetic acid (Fig. 7C). In contrast, we found that eosinophils were more apparent without acetic acid because the natural reflectivity of the eosinophils appeared to be much higher than the surrounding squamous mucosa (Fig. 5C). However, the acetic acid appeared to penetrate the tissue irregularly. This heterogeneous distribution of the contrast manifested in clear visualization of nuclei in some areas (Fig. 6A and C), yet in other areas of the image, nuclei were not as clearly seen (Fig. 7A, arrowhead). One possible cause of the irregular acetic acid penetration might be nonuniform removal of mucus during saline solution washing of the sample. For acetic acid staining to become a viable technique for microscopic contrast in vivo, standard methods for attaining uniform staining must be developed.

The images obtained with SECM are inherently transverse images compared with the conventional cross-sectional images used for histopathology. Here, we acquired multiple transverse images as a function of depth. However, cross-sectional reformatting of the data does not produce high-quality images because the axial resolution of our system $(\sim 10 \mu \mathrm{m})$ was lower than the transverse resolution $(2 \mu \mathrm{m})$. Furthermore, scattering near the top of the sample shadowed deeper images, making the interpretation of images at depths greater than $100 \mu \mathrm{m}$ difficult. These problems may be improved with the development of optimized imaging optics and image-processing routines for correcting attenuation as a function of depth. $^{31,32}$

The number of samples for each mucosal type was not large enough to test the sensitivity and specificity. However, for some types of mucosae, certain distinctive architectural and cellular features were universally observed in most of the SECM images, as shown in the cases of BE with SIM. 
An endoscope-compatible probe must be developed to conduct comprehensive microscopy with SECM in vivo. We have made progress in this area by demonstrating the key components in a bench-top setup. ${ }^{33}$ Figure 8 shows a schematic of the design. Like comprehensive esophageal OFDI, this probe conducts confocal microscopy through a balloon-centering catheter. On the bench top, we have shown that we can acquire SECM images of a $2.5-\mathrm{cm}$ long, $2.0-\mathrm{cm}$ diameter esophageal phantom in less than 2 minutes. ${ }^{33}$ Balloon-catheter SECM is more challenging than OFDI because the axial location of the focus must be controlled to within $10-\mu \mathrm{m}$ precision. Our research developing a comprehensive esophageal SECM probe is currently directed toward finding robust adaptive focusing solutions to address this challenge.

The imaging speed of SECM demonstrated in this study was limited predominantly by the speed of the scanning stages. The endoscopic SECM probe that we are currently developing will provide image information an order of magnitude faster than that in the current study. This balloon-catheter device will conduct rapid scanning by using a high-speed wavelengthswept source and will helically scan the internal optics, in a manner similar to that of OFDI $^{14}$ and our bench-top proof-of-principle SECM probe. ${ }^{33}$

On the completion of the endoscopic probe development, use of SECM will be implemented in patients to provide this detailed diagnostic information over large areas and possibly even the entire distal esophagus. The large-area microscopic imaging enabled by SECM opens up the possibility of comprehensive high-resolution screening for pathology that cannot be identified by conventional video endoscopy and may be missed by confocal endomicroscopy.

\section{Abbreviations}

$\begin{array}{ll}\text { BE } & \text { Barrett's esophagus } \\ \text { H\&E } & \text { hematoxylin and eosin } \\ \text { HGD } & \text { high-grade dysplasia } \\ \text { MGH } & \text { Massachusetts General Hospital } \\ \text { OFDI } & \text { optical frequency domain imaging } \\ \text { RCM } & \text { reflectance confocal microscopy } \\ \text { SECM } & \text { spectrally encoded confocal microscopy } \\ \text { SIM } & \text { specialized intestinal metaplasia }\end{array}$

\section{References}

1. Wong Kee Song L-M, Wilson BC. Endoscopic detection of early upper GI cancers. Best Pract Res Clin Gastroenterol. 2005; 19:833-56. [PubMed: 16338645]

2. Dacosta RS, Wilson BC, Marcon NE. New optical technologies for earlier endoscopic diagnosis of premalignant gastrointestinal lesions. J Gastroenterol Hepatol. 2002; 17(Suppl):S85-104. [PubMed: 12000596]

3. Kiesslich R, Goetz M, Neurath MF. Virtual histology. Best Pract Res Clin Gastroenterol. 2008; 22:883-97. [PubMed: 18790437]

4. Nguyen NQ, Leong RW. Current application of confocal endomicroscopy in gastrointestinal disorders. J Gastroenterol Hepatol. 2008; 23:1483-91. [PubMed: 18761561]

5. Polglase AL, McLaren WJ, Skinner SA, et al. A fluorescence confocal endomicroscope for in vivo microscopy of the upper- and the lower-GI tract. Gastrointest Endosc. 2005; 62:686-95. [PubMed: 16246680] 
6. Kiesslich R, Gossner L, Goetz M, et al. In vivo histology of Barrett's esophagus and associated neoplasia by confocal laser endomicroscopy. Clin Gastroenterol Hepatol. 2006; 4:979-87. [PubMed: 16843068]

7. Muldoon TJ, Anandasabapathy S, Maru D, et al. High-resolution imaging in Barrett's esophagus: a novel, low-cost endoscopic microscope. Gastrointest Endosc. 2008; 68:737-44. [PubMed: 18926182]

8. Pohl H, Rosch T, Vieth M, et al. Miniprobe confocal laser microscopy for the detection of invisible neoplasia in patients with Barrett's esophagus. Gut. 2008; 57:1648-53. [PubMed: 18755886]

9. Becker V, Vercauteren T, von Weyhern $\mathrm{CH}$, et al. High-resolution miniprobe-based confocal microscopy in combination with video mosaicing (with video). Gastrointest Endosc. 2007; 66:1001-7. [PubMed: 17767932]

10. Falk GW, Rice TW, Goldblum JR, et al. Jumbo biopsy forceps protocol still misses unsuspected cancer in Barrett's esophagus with high-grade dysplasia. Gastrointest Endosc. 1999; 49:170-6. [PubMed: 9925694]

11. Loewke, K.; Camarillo, D.; Piyawattanametha, W., et al. Real-time image mosaicing with a handheld dual-axes confocal microscope. In: Tearney, GJ.; Wang, TD., editors. Endoscopic microscopy III; Proceedings of the SPIE; 2008. p. 68510F-9.

12. Cameron AJ, Kamath PS, Carpenter HC. Barrett's esophagus, the prevalence of short and long segments in reflux patients. Gastroenterology. 1995; 108:A65-A.

13. Vakoc BJ, Shishko M, Yun SH, et al. Comprehensive esophageal microscopy by using optical frequency-domain imaging (with video). Gastrointest Endosc. 2007; 65:898-905. [PubMed: 17383652]

14. Suter MJ, Vakoc BJ, Yachimski PS, et al. Comprehensive microscopy of the esophagus in human patients with optical frequency domain imaging. Gastrointest Endosc. 2008; 68:745-53. [PubMed: 18926183]

15. Yoshida S, Tanaka S, Hirata M, et al. Optical biopsy of GI lesions by reflectance-type laserscanning confocal microscopy. Gastrointest Endosc. 2007; 66:144-9. [PubMed: 17591488]

16. Nakao M, Yoshida S, Tanaka S, et al. Optical biopsy of early gastroesophageal cancer by catheterbased reflectance-type laser-scanning confocal microscopy. J Biomed Opt. 2008; 13:054043. [PubMed: 19021423]

17. Collier T, Guillaud M, Follen M, et al. Real-time reflectance confocal microscopy: comparison of two-dimensional images and three-dimensional image stacks for detection of cervical precancer. J Biomed Opt. 2007; 12:024021. [PubMed: 17477736]

18. Sung KB, Richards-Kortum R, Follen M, et al. Fiber optic confocal reflectance microscopy: a new real-time technique to view nuclear morphology in cervical squamous epithelium in vivo. Opt Express. 2003; 11:3171-81. [PubMed: 19471442]

19. Huzaira M, Rius F, Rajadhyaksha M, et al. Topographic variations in normal skin, as viewed by in vivo reflectance confocal microscopy. J Invest Dermatol. 2001; 116:846-52. [PubMed: 11407970]

20. Rajadhyaksha M, González S, Zavislan JM, et al. In vivo confocal scanning laser microscopy of human skin II: advances in instrumentation and comparison with histology. J Invest Dermatol. 1999; 113:293-303. [PubMed: 10469324]

21. Campo-Ruiz V, Patel D, Anderson RR, et al. Virtual biopsy of the joint tissues using near-infrared, reflectance confocal microscopy. A pilot study. Microsc Res Tech. 2006; 69:794-8. [PubMed: 16758475]

22. Tilli MT, Cabrera MC, Parrish AR, et al. Real-time imaging and characterization of human breast tissue by reflectance confocal microscopy. J Biomed Opt. 2007; 12:051901. [PubMed: 17994884]

23. Rajadhyaksha M, Zavislan JM. Confocal laser microscope images tissue in vivo. Laser Focus World. 1997; 33:119.

24. Tearney GJ, Webb RH, Bouma BE. Spectrally encoded confocal microscopy. Opt Lett. 1998; 23:1152-4. [PubMed: 18087457]

25. Boudoux C, Yun S, Oh W, et al. Rapid wavelength-swept spectrally encoded confocal microscopy. Opt Express. 2005; 13:8214-21. [PubMed: 19498851]

26. Wu TT, Qu J, Cheung TH, et al. Study of dynamic process of acetic acid induced-whitening in epithelial tissues at cellular level. Opt Express. 2005; 13:4963-73. [PubMed: 19498484] 
27. Guelrud M, Herrera I, Essenfeld H, et al. Enhanced magnification endoscopy: a new technique to identify specialized intestinal metaplasia in Barrett's esophagus. Gastrointest Endosc. 2001; 53:559-65. [PubMed: 11323579]

28. Hoffman A, Kiesslich R, Bender A, et al. Acetic acid-guided biopsies after magnifying endoscopy compared with random biopsies in the detection of Barrett's esophagus: a prospective randomized trial with crossover design. Gastrointest Endosc. 2006; 64:1-8. [PubMed: 16813794]

29. Sankaranarayanan R, Wesley R, Somanathan T, et al. Visual inspection of the uterine cervix after the application of acetic acid in the detection of cervical carcinoma and its precursors. Cancer. 1998; 83:2150-6. [PubMed: 9827719]

30. Togashi K, Hewett DG, Whitaker DA, et al. The use of acetic acid in magnification chromocolonoscopy for pit pattern analysis of small polyps. Endoscopy. 2006; 38:613-6. [PubMed: 16612744]

31. Wu HX, Ji L. Fully automated intensity compensation for confocal microscopic images. J Microsc. 2005; 220:9-19. [PubMed: 16269059]

32. Capek M, Janacek J, Kubinova L. Methods for compensation of the light attenuation with depth of images captured by a confocal microscope. Microsc Res Tech. 2006; 69:624-35. [PubMed: 16741977]

33. Yelin D, Boudoux C, Bouma BE, et al. Large area confocal microscopy. Opt Lett. 2007; 32:11024. [PubMed: 17410249] 


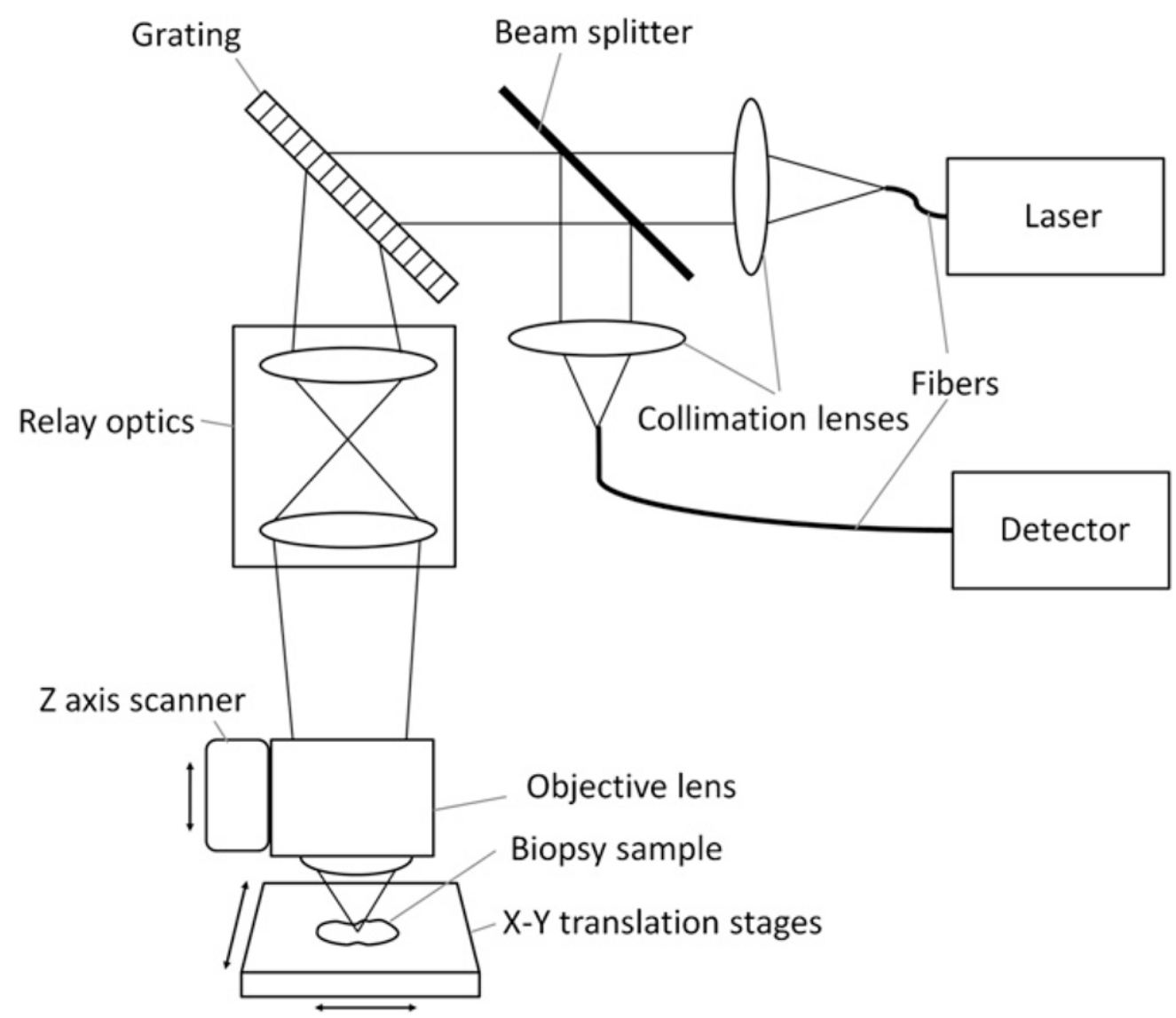

Figure 1.

Schematic of the SECM bench-top system. 

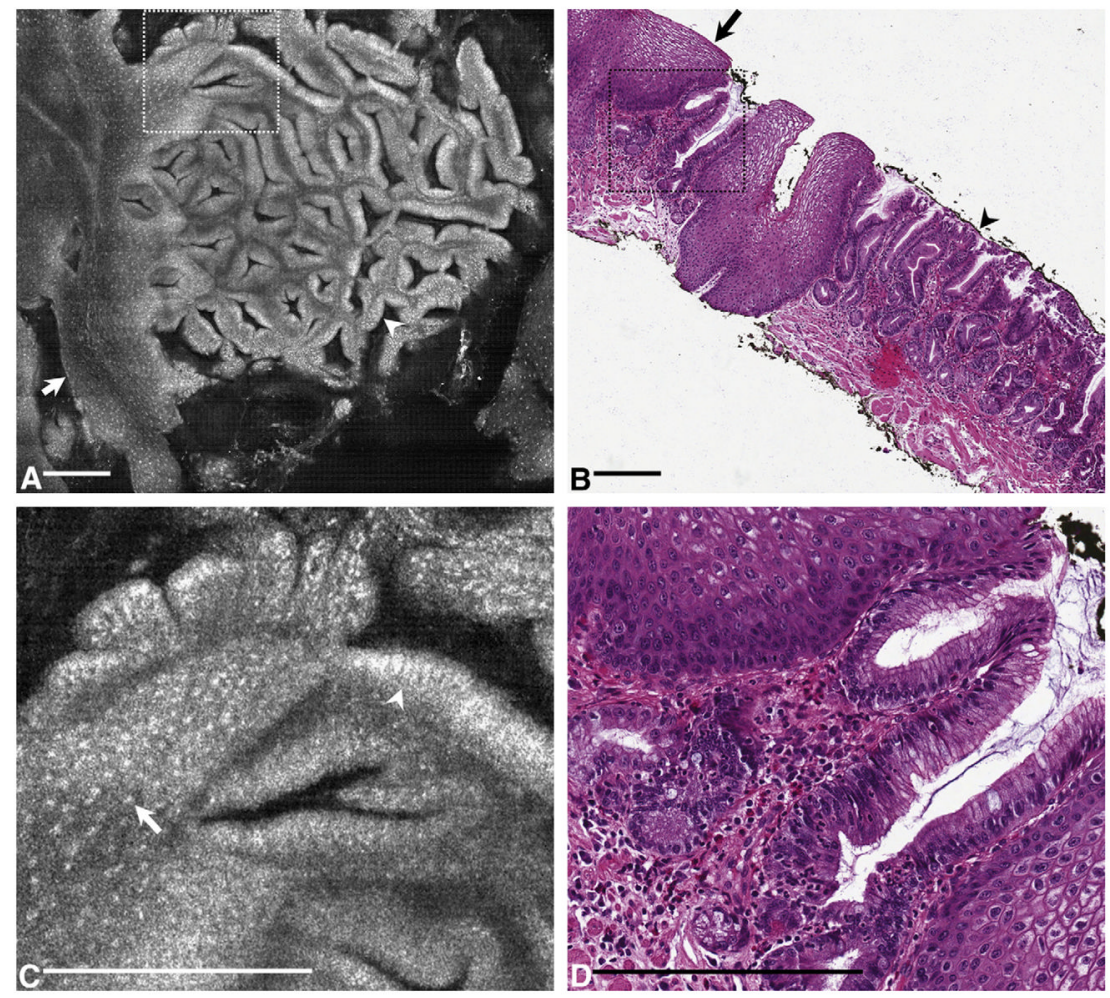

Figure 2.

SECM and histopathologic images of gastroesophageal junctional mucosa stained with $0.6 \%$ acetic acid. A, Large-area SECM image distinguishes gastric cardia type mucosa (arrowhead) from squamous esophageal mucosa (arrow). B, Histopathologic image demonstrates squamoglandular junctional mucosa. C, High-magnification SECM image identified nuclei of columnar epithelial cells (arrowhead) from squamous cells (arrow). D, High-magnification histopathologic image demonstrates different cell types in the stomach and esophagus. Scale bars represent $250 \mu \mathrm{m}$. 

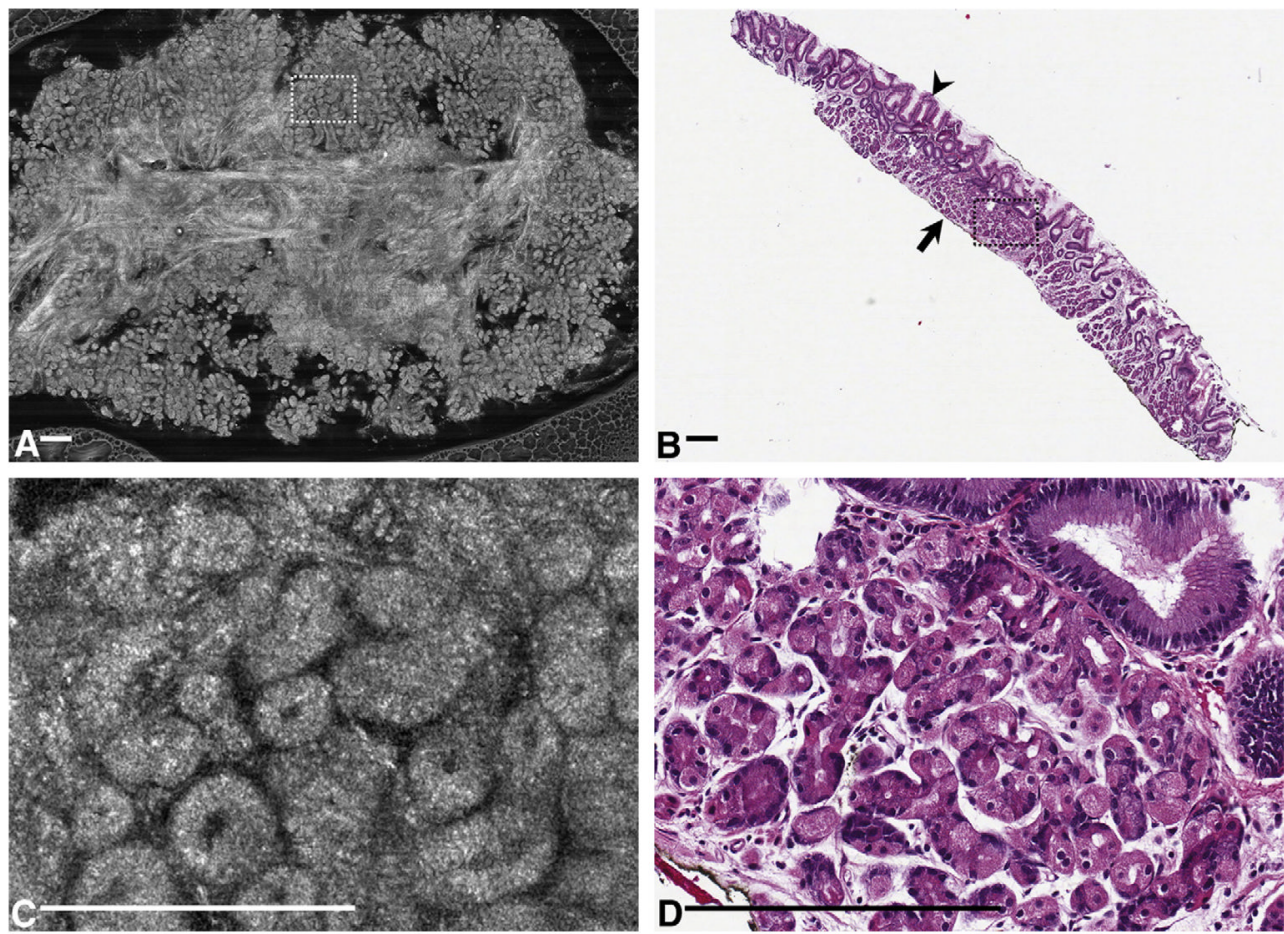

Figure 3.

SECM and histopathologic images of gastric fundic/body type mucosa stained with $0.6 \%$ acetic acid. A, Large-area SECM image shows tightly packed glands. B, Histopathologic image reveals gastric fundic/body type glands (arrow) and foveolar epithelium (arrowhead). C, High-magnification SECM image shows closer look of gastric glands with basally located nuclei. D, High-magnification histopathologic image demonstrates gastric fundic/ body type glands. Scale bars represent $250 \mu \mathrm{m}$. 



Figure 4.

SECM and histopathologic images of a fundic gland polyp stained with $0.6 \%$ acetic acid. A, Large-area SECM image shows irregular glands. B, Histopathologic image also identifies glands with irregular sizes and shapes. $\mathbf{C}$, High-magnification SECM image shows cystically dilated glands lined with cuboidal epithelium. D, High-magnification histopathologic image demonstrates a cellular proliferation of gastric body/fundic type glands with scattered cystically dilated glands consistent with a fundic gland polyp. Scale bars represent $250 \mu \mathrm{m}$. 

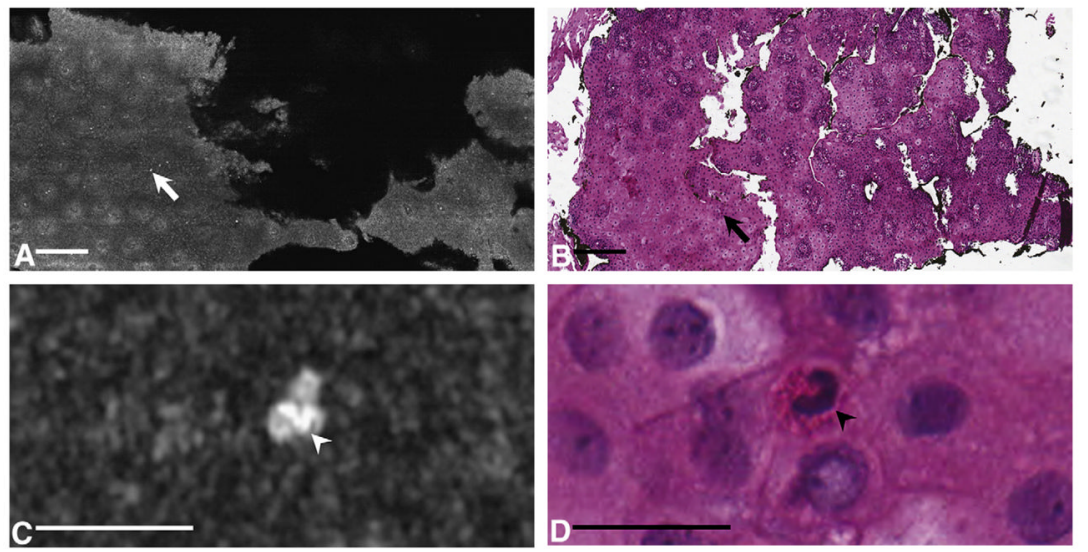

Figure 5.

SECM and histopathologic images of squamous mucosa with scattered eosinophils. A, Large-area SECM image reveals numerous scattered white dots (arrow). B, Histopathologic image demonstrates squamous epithelium with scattered eosinophils. C, High-magnification SECM image of a white dot (arrow in A) shows the characteristic bilobed nucleus (arrowhead) of an eosinophil. D, High-magnification histopathologic image of an eosinophil also shows a bilobed nucleus (arrowhead). Scale bars in A and $\mathbf{B}$ represent $200 \mu \mathrm{m}$ and scale bars in $\mathbf{C}$ and $\mathbf{D}$ represent $20 \mu \mathrm{m}$. 

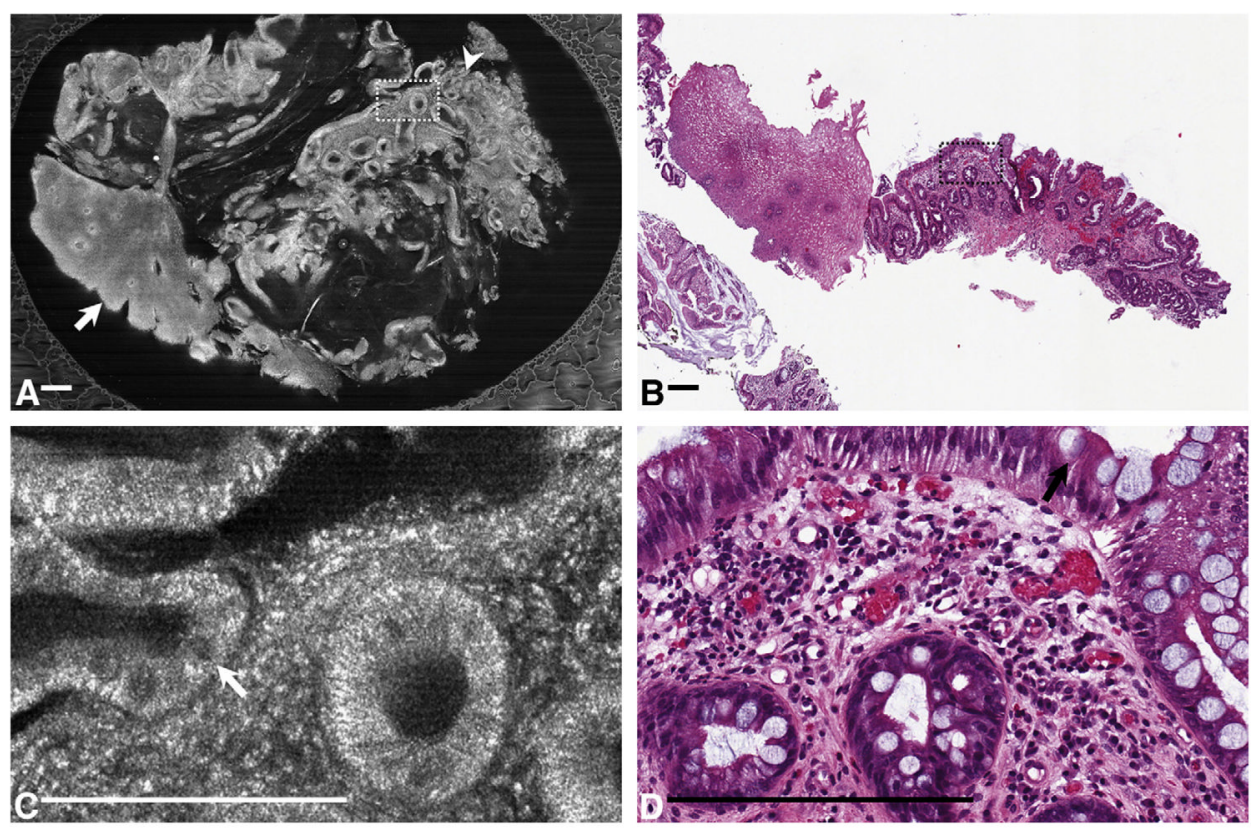

Figure 6.

SECM and histopathologic images of BE stained with $0.6 \%$ acetic acid. A, Large-area SECM image shows columnar epithelium (arrowhead) and squamous epithelium (arrow). B, Histopathologic image demonstrates squamoglandular junctional mucosa. C, Highmagnification SECM image shows the presence of goblet cells (arrow). D, Highmagnification histopathologic image shows BE with the presence of goblet cells (arrow). Scale bars represent $250 \mu \mathrm{m}$. 

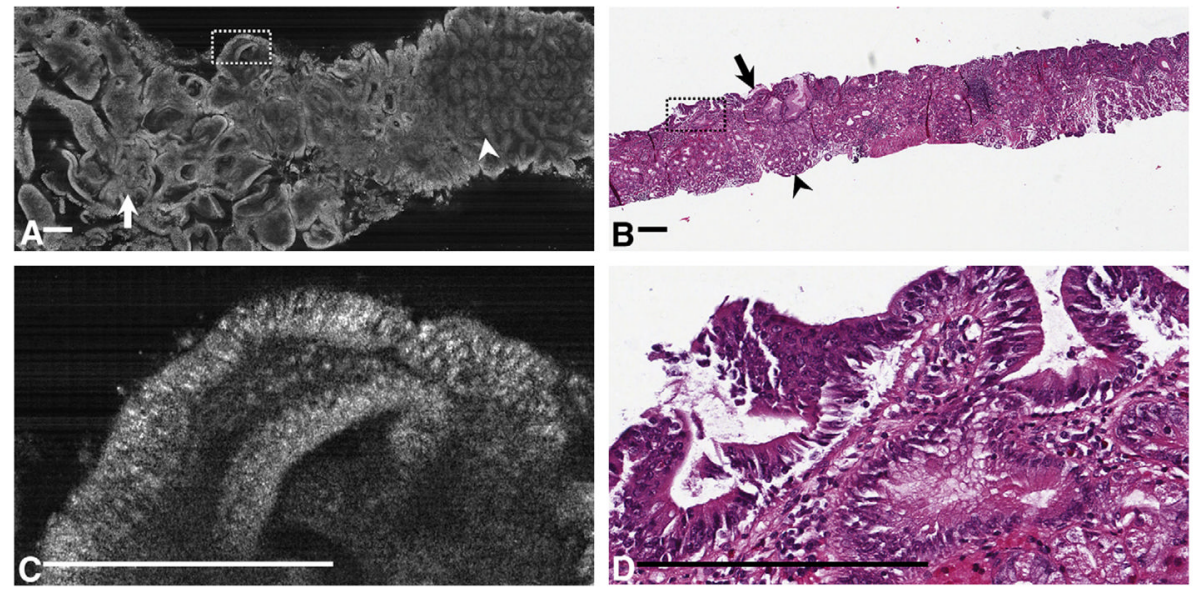

Figure 7.

SECM and histopathologic images of BE with dysplasia stained with $0.6 \%$ acetic acid. A, Large-area SECM image shows mixture of disorganized glands (arrow) and normalappearing glands (arrowhead). B, Histopathologic image demonstrates dysplastic surface epithelium (arrow) and regularly arranged glands (arrowhead). C, High-magnification SECM image shows the loss of nuclear polarity in the dysplastic epithelium. D, Highmagnification histopathologic image also depicts nuclear atypia. Scale bars represent 250 $\mu \mathrm{m}$. 


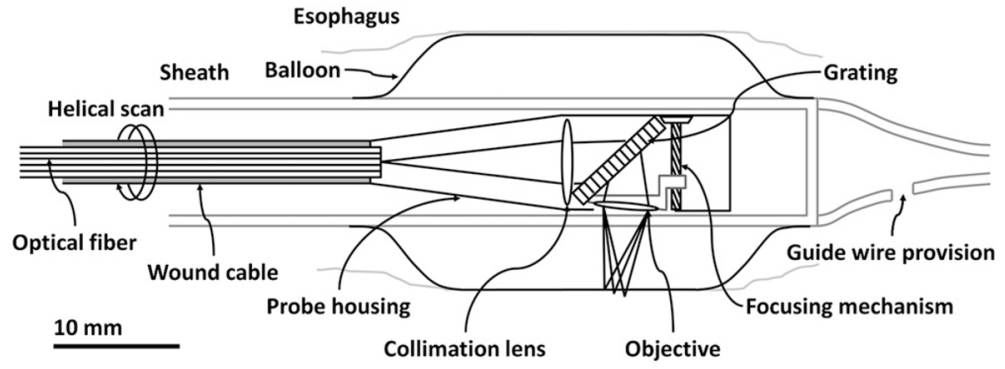

Figure 8.

Schematic of a SECM endoscopic probe design for conducting comprehensive confocal microscopy of the esophagus. 
TABLE 1

Diagnoses of correctly oriented biopsies

\begin{tabular}{|lc|}
\hline Mucosa type & No. biopsies (no. acetic acid-stained biopsy samples) \\
\hline Squamous mucosa & $13(7)$ \\
GEJ mucosa & $11(8)$ \\
Gastric antral mucosa & $2(1)$ \\
Gastric fundic/body type & $2(2)$ \\
Fundic gland polyp & $1(1)$ \\
BE, no dysplasia & $6(6)$ \\
BE, dysplasia & $2(1)$ \\
Total & $37(26)$ \\
\hline
\end{tabular}

$G E J$, Gastroesophageal junction; $B E$, Barrett's esophagus. 